\title{
Update on pediatric hip imaging
}

Nele Herregods ${ }^{1} \mathrm{MD}$, PhD; Lennart Jans ${ }^{1} \mathrm{MD}$, PhD; Jacob Lester Jaremko ${ }^{2} \mathrm{MD}$, PhD; Filip M. Vanhoenacker ${ }^{3} \mathrm{MD}, \mathrm{PhD}$.

From:

1. Department of Radiology and Medical Imaging Ghent University Hospital

De Pintelaan 185

9000 Gent

Belgium

2. Department of Radiology \& Diagnostic Imaging

University of Alberta Hospital

8440-112 Street

Edmonton T6G 2B7, Alberta

Canada

3. Department of Radiology and Nuclear Medicine

University Hospital Antwerp

Wilrijkstraat 10

2650 Antwerp (Edegem)

Belgium

Corresponding author:

Nele Herregods, MD, PhD.

Ghent University Hospital

De Pintelaan 185

9000 Gent

Belgium

Tel: 0032/9.332.66.70

Fax: 0032/9.332.49.69

Nele.Herregods@uzgent.be 


\begin{abstract}
Hip disorders are common in children. Prompt diagnosis and treatment are important because of the potential complications. Symptoms are frequently nonspecific (hip pain, limp, irritability), and clinical examination can be difficult and unreliable, especially in smaller children. Therefore, imaging can be valuable. Imaging modalities available for evaluation of the hip include radiography (RX), ultrasound (US), Computed Tomography (CT), Magnetic Resonance Imaging (MRI) and bone scintigraphy.

Radiography and ultrasound remain the initial imaging modalities of choice. Increasingly, MRI is obtained for assessing the pediatric hip, although the long imaging time and need for sedation may limit its use in daily clinical practice. Because of the exposure to ionizing radiation, the use of CT and bone scintigraphy in children is limited to selected cases.

Pediatric hip pathology varies depending on patient age. This article provides an overview of the common hip pathologies in children, including congenital and developmental pathologies, trauma, infectious processes, inflammatory disease and neoplasm. The age of the child, history and clinical examination are essential to narrow down the differential diagnosis and subsequent selection of the appropriate imaging modality.
\end{abstract}

Keywords: Hip, pediatric, imaging, pain 


\section{Introduction}

Hip disorders are highly prevalent in children. Accurate diagnosis and treatment are important because of the potential complications, which may leed to long term morbidity such as degenerative joint disease in adult life. Diseases of the hip in children can cause nonspecific symptoms such as groin pain, limping, or pain radiating into the thigh and/or knee. Clinical examination can be difficult in children, especially in smaller children, and may be unreliable. The age of the child and the duration of the symptoms are important to make a differential diagnosis. In children with symptoms less than 5 days, without fever or elevated inflammatory markers, a wait-and-see policy is recommended. Otherwise, as the diagnosis is often not clear, imaging can be of value. Imaging findings should be interpreted together with clinical history, physical examination and laboratory findings to prompt an accurate diagnosis.

The hip of the child is not a "miniature adult hip". In hips that are not yet mature, the structures which later will result in the neck, head and greater trochanter, still partially exist of cartilage that progressively ossifies. By the age of 12 , the greater trochanter and the femoral head are completely ossified. The growth plate of the femoral head is common to the greater trochanter and remains active, only closing after puberty. The blood perfusion of the femoral head in children depends solely on an arterial anastomotic network in the posterior region of the femoral neck. There is no flow to the femoral head due to the presence of a growth plate. There is growth in the transition area from the head to the femoral neck (growth cartilage), responsible for normal growth of the femoral head and neck lengthening, growth cartilage in the greater trochanter, and the triradiate growth cartilage of the acetabulum that grows in depth and width so as to maintain its sphericity concave and lodge the femoral head. Lesion or trauma of this regions may predispose tot severe anomalies in the development of the femoral head or deformities of the acetabulum.

In this article we provide an overview of the common hip pathologies in children, including congenital and developmental pathologies, trauma, infectious processes, inflammatory causes and neoplasia (benign or malign) (Table 1).

\section{Imaging modalities}

The imaging modalities available for evaluation of the hip are conventional radiography (CR), ultrasound (US), Computed Tomography (CT), Magnetic Resonance Imaging (MRI) and bone scintigraphy.

\section{Conventional Radiography}

Conventional Radiography $(\mathrm{CR})$ remains the first choice imaging modality. Because the appearance of a child's hips varies with age, consideration should be given to obtain anteroposterior and frog-leg view (hips externally rotated and flexed) images of the entire pelvis rather than views of a single hip [goughpalmer]. This allows easy comparison with a 'normal' hip. Gonad shielding during girls pelvic x-ray is no longer indicated. However in boys it is controversial and depends on the skill and effort of radiographers [Karami, Frantzen]. 


\section{Ultrasound}

Ultrasonography (US) is also imaging modality of choice, especially for identifying hip joint effusion, for guiding fluid aspiration and for assessment of the unossified structures. US has low cost, does not involve ionizing radiation, is readily available, and allows dynamic evaluation. Disadvantage is its highly operator dependence.

\section{Computed tomography}

Computed tomography (CT) has limited indications for the assessment of the pediatric hip because of the exposure to ionizing radiation. CT may be indicated for preoperative assessment of developmental dysplasia of the hip (DDH), Legg-Calvé-Perthes or slipped capital femoral epiphysis (SCFE), evaluation of tumor matrix or cortical invasion, and guidance for biopsy or ablation.

Magnetic Resonance Imaging

Magnetic Resonance Imaging (MRI) is increasingly obtained. MRI is excellent for detecting early disease and can depict not only bone marrow edema (BME), but also soft tissue inflammation and joint effusion/bursitis, as it had very good soft tissue contrast resolution. The cartilage can be assessed as well. Disadvantages are the relatively higher cost, the accessibility and the need for sedation in younger children.

Bone scintigraphy

Bone scintigraphy can evaluate a large area, but is often non-specific and has exposure to ionizing radiation. It may be indicated for evaluation of osteomyelitis, Legg-Calvé-Perthes, osteoid osteoma, stress fractures or metastatic disease when MRI is contra-indicated.

Table 1: Common disease of the pediatric hip

\section{Congenital/developmental Developmental dysplasia of the hip}

Legg-calvé-Perthes disease

Coxa vara

Neuromuscular hip dysplasia

Traumatic

Slipped capital femoral epiphysis

(stress) Fracture

Apophyseal injury

Stress reactions at the ischiopubic synchondrosis

Infectious

Septic Arthritis

Osteomyelitis/CRMO

\section{Inflammatory}

Transient Synovitis

Juvenile Idiopathic Arthritis 


$\begin{array}{ll}\text { Neoplastic } & \text { Benign } \\ & \text { Malignant }\end{array}$

\section{A. Congenital/developmental}

\section{A.1. Developmental dysplasia of the hip}

Developmental dysplasia of the hip (DDH) is a relatively common disorder that involves abnormal development of the femoral head and acetabulum. It is more common in girls, in Caucasians, after breech deliveries and with a positive family history. The incidence ranges from 1.5 to 20 per 1000 births. It consists of a spectrum of abnormalities from birth to early infancy ranging from a stable hip with mild dysplasia to complete hip dislocation [bracken]. Early detection is mandatory to prevent labral tears and cartilage lesions at young age and development of premature degenerative hip disease. Different screening strategies have been described, including clinical examination alone, and selective - in patients with associated risk factors or abnormal clinical examinations - or universal ultrasound screening [ortiz]. Physical examination classically involves the Barlow and Ortolani maneuvers [giannakopoulou]. Ultrasonography (US) is still the imaging modality of choice for infants below the age of four-five months [bracken1-2]. In children older than five-six months, conventional radiography (CR) is indicated as progressing ossification of the femoral head prevents adequate evaluation with ultrasound [Gill, starr].

The American College of Radiology recommends that for a standard ultrasound examination two orthogonal planes should be performed: a coronal view in the standard plane at rest and a transverse view of the flexed hip with and without stress, and Graf $\alpha$ and $\beta$ angles are measured [American institute]. The modified Graf grading classification (4 types - Table 2 ) is based on the $\alpha$ angle and degree of acetabular roof coverage [graf, starr]. To determine the position and stability of the femoral head, dynamic stress imaging could be performed, similar to the clinical Barlow examination. When monitoring is performed in the Pavlik harness, the infant is left in the harness and only static images are obtained [el ferzli]. Color Doppler imaging may be used to evaluate perfusion to the proximal femoral epiphysis, although there is little published on this topic [Barnewallt].

Table 2: Modified Graf Classification Scale [graf]

\begin{tabular}{|c|c|c|}
\hline Graf Type & Description & $\alpha$ and $\beta$ Angle \\
\hline Type 1 & $\begin{array}{l}\text { Normal, mature hip with more than } 50 \% \text { acetabular } \\
\text { roof coverage }\end{array}$ & $\alpha$ angle $\geq 60^{\circ}, \beta$ angle $<55^{\circ}$ \\
\hline Type 2a & Physiologic immaturity at younger than 3 mo & $\alpha$ angle $50-59^{\circ}$ \\
\hline Type $2 b$ & Immature at age 3 mo or older & $\alpha$ angle $50-59^{\circ}$ \\
\hline Type $2 \mathrm{c}$ & $\begin{array}{l}\text { Extremely deficient bony acetabulum; femoral head } \\
\text { is concentric but not stable }\end{array}$ & $\alpha$ angle $43-49^{\circ}, \beta$ angle $<77^{\circ}$ \\
\hline Type 2d & $\begin{array}{l}\text { Femoral head is grossly subluxed and labrum is } \\
\text { everted, increasing } \beta \text { angle }\end{array}$ & $\begin{array}{l}\alpha \text { angle difficult to measure but is } \\
\text { approximately } 43-49^{\circ} ; \beta \text { angle }>77^{\circ}\end{array}$ \\
\hline Type 3 & Dislocated femoral head with shallow acetabulum & $\alpha$ angle $<43^{\circ}$ \\
\hline Type 4 & Dislocated femoral head with severely shallow, & \\
\hline
\end{tabular}


dysplastic acetabulum and inverted labrum

For radiographic evaluation, an anteroposterior radiograph of the hips in neutral position is obtained. Familiarity with the normal growth and development is essential. A frog-leg lateral view is sometimes used to determine whether a subluxed hip reduces. Several lines and angles are used to diagnose and further characterize DDH: the Hilgenreiner, Perkins and Shenton line, and the acetabular and centeredge angle. In $\mathrm{DDH}$, there is delayed ossification of the femoral head and an abnormally shallow acetabulum, predisposing to subluxation and dislocation [Donnelly, beltran]. Additionally, late complications, such as osteoarthritis and avascular necrosis, can be assessed too [starr 2, Donnelly, beltren].

CT with three-dimensional images may be used for preoperative planning or postoperative evaluation of reduction when MRI is contra-indicated or not available [murray]; reduced-dose CT-protocols should be used.

Both imaging modalities have excellent sensitivity and specificity for the evaluation of hip reduction, but CT has the disadvantage of radiation exposure, however, it has shorter imaging time and therefore needs less sedation [chin]. For the MRI protocol, axial and coronal Short-tau Inversion Recovery (STIR) or T2weighted sequences with or without corresponding T1-weighted sequences can be obtained [starr, McNally], some studies even suggest that an axial STIR sequence alone may be sufficient [conroy, laor]. However, if there is concern for avascular necrosis of the femoral head, gadolinium - sequences are used to evaluate for femoral head enhancement abnormalities [Jaramillo, Tiderius]. MRI depicts the unossified acetabular epiphysis in the ilium and underlying labrum, therefore showing greater and more accurate acetabular coverage than seen on radiography alone [Kim]. Recent orthopedic articles have described the utility of both bony and cartilaginous acetabular indexes on MRI in the evaluation of DDH [Li, pirpiris]. After successful reduction, the femoral head should be concentrically located in the acetabulum [ray].

Jacob: 3D US?

\section{A.2. Legg-Calvé-Perthes disease}

Perthes or Legg-Calve-Perthes (LCP) disease is an idiopathic avascular necrosis (AN) of the proximal femoral epiphysis in children [gill]. It occurs more commonly in boys, typically between the age of $4-8$ years, but may range from 2 years of age untill adolescence. Bilateral involvement may occur in $10-20 \%$, but usually it is seen unilaterally [gill]. The pathogenesis of Perthes disease is not completely understood and possibly related to a transient disturbance of the blood supply via the lateral epiphyseal vessels, resulting in ischemia and infarction, or trauma [Gill,de smet]. Although approximately $60-70 \%$ of children with LCP heal spontaneously without functional impairment, late onset in children older than 8 year, and nonspherical femoral head at skeletal maturity are associated with a higher incidence of premature osteoarthritis before the fifth decade of life. Children may present with limping and hip pain, aggravating by exercise and often irradiating to the knee, or may refuse to walk (younger children) [de smet, kocher].

Radiography is the imaging modality of choice, and may show fragmentation and flattening of the sclerotic capital epiphysis, features best appreciated on the frog-leg lateral view. During the course of the disease, metaphyseal widening and cysts may occur as well as femoral head enlargement. 
Radiographic changes vary based on the stage of the disease and are summarized in Figure 1 [Vanhoenacker,Gill]. Multiple radiographic classification systems are described, of which the most common are the Catterall classification and the Herring classification, of which the latter is most accurate in predicting long-term prognosis (figure 2) [herring, vanhoenacker].

US is unlikely to pick up LCP, although a joint effusion can be seen.

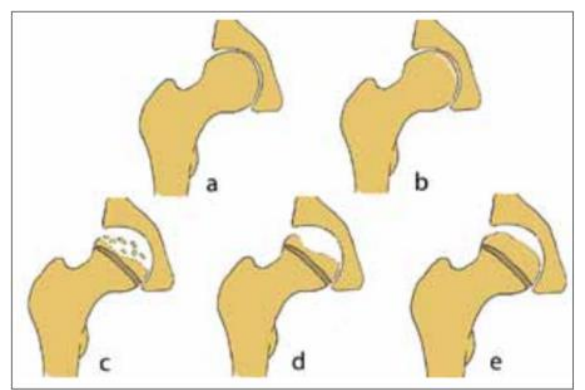

Figure 1: Schematic drawing of the radiographic changes based on the stage of the disease [gill, vanhoenacker].

(a) Stage I, no visible radiographic abnormalities. (b) Stage II, early: subchondral crescent line. (c) Stage II, late: collapse, decreased height and fragmentation of the epiphysis. (d) Stage III, regeneration phase with beginning reossification and remodeling. (e) Stage IV, complete healing.

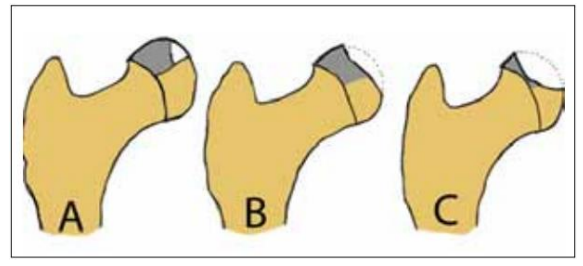

Figure 2: Herring classification, based on the degree of collaps of the lateral epiphyseal pillar [herring, vanhoenacker].

Stage A: Normal contour of the lateral pillar, indicating a good prognosis. Stage B: Decrease of height of the lateral pillar of less than $50 \%$. The prognosis is usually good in patient of less than 6 years old, the prognosis is variable in the older age group. Stage $C$ : Decrease of height of the lateral pillar of more than $50 \%$, indicating a bad prognosis.

If the X-ray is normal and persistent symptoms trigger further imaging, MRI can be performed. MRI may show proximal femoral abnormalities before radiography, and can also assess revascularization, healing and complications [Dillman]. On T1-weighted images, the proximal femoral ossification center will show decreased signal intensity. The signal on T2-weighted images is variable. Areas of high signal correspond to bone marrow edema, whereas areas of low signal on $\mathrm{T} 1$ and $\mathrm{T} 2$ sequences suggest advanced necrosis. Curvilinear subchondral T2 hyperintensity corresponds to subchondral fractures ("crescent sign"). A mild joint effusion and synovitis are often present. The overlying articular cartilage may appear thickened. It is important to obtain sagittal images as well, as the disease process often begins anteriorly, which may be missed on coronal and axial images [gill]. Hypoperfusion, seen as absent or asymmetrically decreased enhancement may be one of the earliest indicators [gill]. On follow-up MRI, reappearance of enhancement of the femoral head epiphysis can be seen, due to revascularization. Early reperfusion of the lateral pillar is associated with a better prognosis [gill]. In the reparative phase, epiphyseal fragments 
may demonstrate different signal characteristics, owing to different stages of healing. In the late stage, femoral head height is restored as these fragments coalesce [gill]. After approximately 6 years, signal intensity returns to normal [cheng].

If $\mathrm{MRI}$ is contraindicated or not available, bone scintigraphy can be helpful, as scintigraphic changes precede radiographic findings. A focal area of decreased uptake is seen [hubbard]. The role of CT is limited.

\section{A.3. Coxa vara}

In coxa vara the angle between the femoral neck and shaft is decreased compared with age-matched normal hips, usually to less than $120^{\circ}$ (Figure 3). The normal femoral neck-shaft angle ranges between $120-135^{\circ}$, and decreases from $150^{\circ}$ in infants to $120^{\circ}$ in adults [hubbard]. Coxa vara deformity results from a failure of medial growth of the physeal plate, and can be unilateral or bilateral. It may be acquired (secondary to prior infection, trauma or avascular necrosis) or true (secondary to congenital abnormalities or developmental). Coxa vara can also be seen in association with syndromes such as osteogenesis imperfect or fibrous dysplasia. Developmental coxa vara usually presents around the age of 2 with an abnormal gait or painless limp. This form may be bilateral in as many as one half of cases, and is thought to be secondary to abnormal cartilage proliferation in the medial physis that causes increased lateral growth, resulting in a more vertical than usual orientation of the physis [bos,serafin, zucker]. Treatment is surgical, although it frequently recurs after surgery as the growth disturbance progresses until complete skeletal maturation [desai, günter].

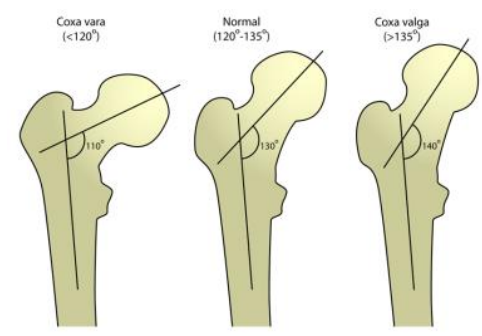

Figure 3: Femoral neck-shaft angle in normal hip, coxa valga and coxa vara.

Conventional radiography and MRI are the imaging modalities of choice. On CR, the femoral neck-shaft angle can be measured. Radiographic changes include a widened, irregular, vertically oriented physis. Due to abnormal stress in the physis, multiple small slips occur and small triangular corner fractures develop along the medial aspect of the physis, seen as metaphyseal bony fragments inferomedial to the physis. MRI can be useful in preoperative planning, and may show physeal widening or bone marrow edema, and can assess potential impingement [zucker].

\section{A.4. Neuromuscular hip dysplasia/ coxa valga}

Children with either increased or decreased muscle tone are at risk for secondary hip dysplasia. Children who do not walk before the age of five have a $58 \%$ chance of hip dislocations, which are bilateral in $44 \%$ of the cases [scrutton1]. Hip dysplasia is also a common problem in children with cerebral palsy. The hip 
is normal at birth, but the lack of physiologic stress results in coxa valga and increased anteversion of the femur. Secondly, posterolateral subluxation may occur due to muscle imbalance resulting in flexion and adduction contractures [chang]. Symptoms include sitting imbalance, walking difficulty, decubitus ulcers from abnormal positioning and pain [khalife]. The acetabulum becomes globally dysplastic, resulting in posterior deficiency. Hip dislocation can be prevented through radiographic screening and early treatment.

Conventional radiography is the imaging modality of choice, showing the valgus deformity, an abnormal shape and location of the femoral head and a dysplastic acetabulum [haglund]. Also, the acetabular index can be measured, as well as the migration percentage (MP), describing the degree of femoral head containment within the acetabulum, or the proportion of the ossified femoral head that lies lateral to the Perkins line [REIMERS]. In normal hips, up to $25 \%$ of the femoral head can be uncovered. Generally, in children with a MP $>33 \%$, a varus derotational osteotomy of the femur is indicated [terjesen, scrutton2]. Postoperatively, radiographic evaluation for signs of redislocation or avascular necrosis is indicated [khalife]. It is important to image the patient in the most neutral position possible, as femoral adduction/abduction gives a false respectively high/low MP.

CT and MRI are helpful preoperatively to evaluate the orientation and shape of the acetabulum, and for assessing the femoral anteverion and tibial torsion.

\section{B. Traumatic}

\section{B.1. Slipped capital femoral epiphysis}

Slipped capital femoral epiphysis (SCFE) is one of the most common hip disorders of adolescence, characterized by an idiopathic Salter Harris type 1 fracture through the proximal femoral physis, resulting in posteromedial displacement of the capital femoral epiphysis. It typically occurs in adolescents between the age of $11-15$ years, more commonly in boys than in girls, and can be acute (symptoms $<3$ weeks) or chronic. Bilateral involvement may occur in 20-30 \% of cases [gholve, zucker]. It is attributed to a variety of conditions, including obesity, metabolic disorders, endocrine disorders and radiation therapy. Estrogen seems to play a protective factor reinforcing the growth plate, which may explain why males are more affected than females [gholve]. Children present with chronic hip, groin or referred knee pain or may limp. Immediate treatment of SCFE is mandatory, consisting of non-weight-bearing therapy and single-screw fixation. Delayed diagnosis and therapy can lead to avascular necrosis (AN), gait abnormalities and premature osteoarthritis, femoroacetabular impingement and leg length discrepancy [Gholve,Tins 1]. AN occurs in $20-45 \%$ of cases [lubicky].

Conventional radiography remain the prime method of diagnosing SCFE. Views of both hips should be obtained, because of the high incidence of bilateral disease. Antero-posterior (AP) as well as frog-leg views are required, as the slip may only be evident on the frog-leg lateral view. Typical findings are widening and irregularity of the physis, but this can easily be overlooked on the AP view. The frog-leg view shows the posteromedial slippage of the femoral epiphysis. Klein's line should be drawn along the lateral femoral neck margin on an AP view, and along the superior margin on the frog-leg view. The line should intersect the lateral aspect of the femoral head in normal subjects, as in SCFE, this line fails to intersect the lateral aspect of the femoral head ("Trethowan's sign"). An increased density in the proximal metaphysis can be seen as well ("metaphyseal blanch sign") [Vanhoenacker book]. 
MRI can play a role in early diagnosis of clinically suspected cases with normal radiographic findings ("preslip SCFE"). In these cases, physeal widening can be seen, BME in the epiphysis or proximal metaphysis, and joint fluid. BME adjacent to the growth plate can be considered as an alarming sign [Tins2]. MRI is also the best imaging modality for detecting complications. MRI allows to examine the contralateral hip as well.

CT is rarely needed for diagnosis, but may be used preoperatively to measure the degree of femoral epiphyseal tilt and the precise relationship of femoral head to the metaphysis in three planes.

\section{B.2. (Stress) fracture}

Acetabular or pelvic ring fractures are less common in children than in adolescents or adults because of the greater elasticity of the pediatric pelvis, particularly if the triradiate cartilage is not yet fully ossified [Johnson, boutry]. Femoral neck fractures are also uncommon in children. These fractures result usually from high energy trauma, but pathological fractures resulting from underlying metabolic disease (osteogenesis imperfecta, osteopetrosis) or focal bony lesions/tumors (e.g. bone cysts) may occur [watts, torode]. Acetabular fractures are often associated with traumatic posterior hip dislocations [quick]. Watts proposed a classification system with recommendations for management of acetabular fractures in children (Table) [watts]. Torode and Zieg classified pediatric pelvic fractures in 4 groups, of which the first three groups are stable with good prognosis, and the fourth type is unstable (figure) [torode]. Pediatric femoral neck fractures are classified by Delbet into 4 groups, of which the first three groups have high risk for AVN, the fourth group has no increased risk for AVN (figure) [quick]. One should be aware of other more rare complications such as non-union, premature fusion of the growth plate, femoral head deformity, arthritic changes and leg length discrepancy [quick, vanhoenacker book].

CT is the preferred method for precise evaluation of the lesion extension and to identify unstable fractures, and also to evaluate associated trauma [quick, vanhoenacker]. For low energy trauma or femoral neck fractures, conventional radiography is usually sufficient [bali]. MRI is indicated in case of suspicion of labral damage or osteochondral fragment [watts, vanhoenacker].

\begin{tabular}{|l|l|l|}
\hline Watts type & Lesion extent & Treatment \\
\hline A & $\begin{array}{l}\text { Small posterior wall fracture fragments due to hip } \\
\text { dislocation }\end{array}$ & $\begin{array}{l}\text { Conservative; MRI in case of suspicion of labral damage } \\
\text { or osteochondral fragment }\end{array}$ \\
\hline B & $\begin{array}{l}\text { Undisplaced linear fracture and associated pelvic } \\
\text { fractures }\end{array}$ & $\begin{array}{l}\text { Conservative; MRI in case of suspicion of labral damage } \\
\text { or osteochondral fragment }\end{array}$ \\
\hline C & Linear fracture with unstable hip & Open reduction and internal fixation \\
\hline D & Central fracture dislocation of the hip & No consensus because high risk AVN \\
\hline
\end{tabular}

Table : Watts classification system and recommendations for acetabular fractures [watts, vanhoenacker book]. 


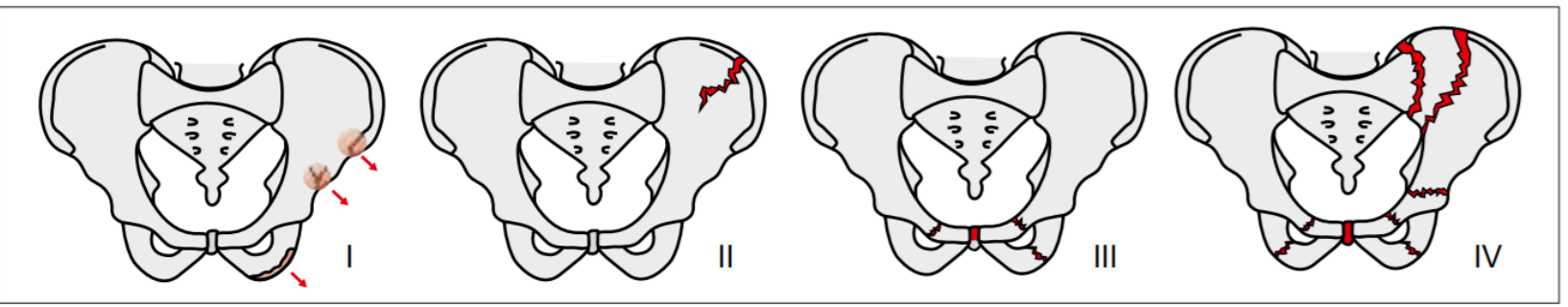

Figure : Torode-Zieg classification of pediatric pelvic fractures [torode]. Groups I-III are stable with good prognosis, type IV is unstable.

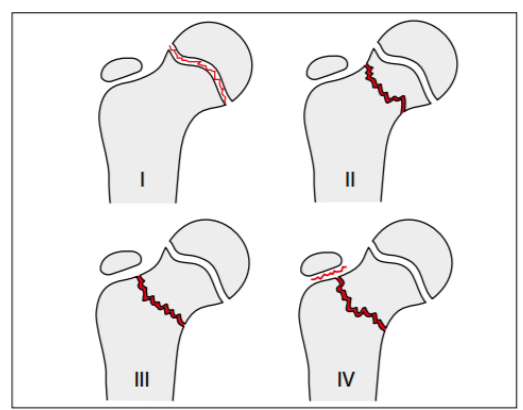

Figure : Delbet classification of pediatric femoral neck fractures [boutry, vanhoenacker]. Groups I-III have high risk for AVN, group IV has no increased risk for AVN.

Stress fractures of the pelvis are caused by abnormal stress on otherwise normal bone. They are relatively uncommon in children and adolescents, and occur mainly in high-level athletes training with repetitive loading activities [karantanas]. Radiographic findings are initially normal in most cases, and can detect typical findings such as periosteal reaction, endostal cortical thickening and a lucent cortical fracture line by 2-14 weeks [Behrens, Campbell]. MRI and bone scintigraphy are approximately equally sensitive for early detection, but MRI is more specific and is therefore the preferred imaging method [Campbell, arendt]. MRI may show the fracture line, but in some cases only periosteal and bone marrow edema are seen, this is more appropriately called a stress reaction.

Finally, one must always keep in mind the possibility of non-accidental trauma, typically metaphyseal 'corner' lesions or fractures in a child not of walking age may be seen.

\section{B.3. Apophyseal injury}

Apophyseal avulsion injuries are Salter Harris type 1 fractures, unique to the growing skeleton. They are a frequent cause of hip pain in adolescents, boys more than girls, particularly those involved in sports. In the immature skeleton, the tendons are generally stronger than the apophyses, and forceful or repetitive traction from the attached muscles can result in an apophyseal avulsion fracture [rossi]. Avulsion fractures of the anterior superior iliac spine (ASIS) and anterior inferior iliac spine mostly occur in soccer players, injuries to the ischial tuberosity (IT) in gymnasts [rossi, vandervliet]. Other apophyses involved are the iliac crest, and the greater and lesser trochanters [kovacevic]. Clinically, sudden pain, exacerbated by activity, followed by restricted motion and weakness may occur [rossi]. Apophyseal 
injuries are usually treated conservatively with rest. In case of avulsion fractures with displacement of more than $2 \mathrm{~cm}$, surgical fixation may be required [vandervliet].

Conventional radiography is usually diagnostic. Radiographs demonstrate displacement of the apophysis from its expected position or bone resorption in the early phase, followed by callus formation and bony reaction. Comparison with the contralateral side may help to distinguish a true avulsion fracture from a normal unfused apophysis. In case of suspected or subtle avulsion with negative radiographs, or in cases where the apophysis has not yet ossified, MRI is useful [stevens, vandervliet, mckinny]. MRI may show high T2 signal in the disrupted physis, as well as in the adjacent bone and soft tissues. T2-hyperintensity, periosteal stripping at the insertion of the tendons and even tendon tears can be seen as well [Meyers]. In the healing phase, fibrous healing may be seen as a focal hypointense bridging of the apophyseal growth plate, distinguishing it from a true non-union, where a persistent high signal intensity can be seen on fat-suppressed (FS) T2-weighted or STIR images between the displaced apophyseal fragment and the underlying bone [vandervliet]. However, comparison with the opposite side is useful as some degree of high T2 signal is expected in the actively growing apophysis. Excessive callus formation may cause impingement, in which case additional CT may be needed for precise evaluation of bony tunnel narrowing if surgical resection is considered [stevens, vansevenant].

In case of chronic repetitive microtrauma, no or little displacement of the physis may occur, and the physis appears widened and irregular on CR. MRI will show marked edema and enhancement in the physis, adjacent muscles and bone marrow, consistent with apophysitis. This is important to recognize, as it can be mistaken for malignancy [laor].

\section{B.4. Stress reactions at the ischiopubic synchondrosis}

The ischiopubic synchondrosis (IPS) is a cartilaginous joint that typically closes before or during early adult life. The ossification of the cartilage between the ischium and the pubis is highly variable, and IPS enlargement may be an incidental radiological finding [karantanas, oliveira]. When seen bilaterally and symmetrically, it causes usually no diagnostic problems. In case of asymmetrical closure of the synchondrosis, particularly in sportive children, it may cause confusion with a bone tumor or osteomyelitis. Ischiopubic synchondrosis asymmetry is much more common on the side of the weightbearing non-dominant foot, with the differences in ossification thought to result from asymmetric mechanical forces [Herneth]. Clinically, there may be groin pain and restriction of movement. Treatment is conservative.

On conventional radiography, a tumour-like radiolucent area at the fusion zone with surrounding sclerosis may be seen. MRI findings are nonspecific and may be misleading, showing enlargement of the involved IPS, edema in the bone and adjacent soft tissues, and enhancement adjacent to the synchondrosis [herneth2]. On bone scintigraphy, increased uptake at the weight-bearing side may be seen.

Recognition of this normal variant is important when interpreting a pediatric pelvic radiograph. If seen asymptomatic without concerning history, it most likely represents a developmental variant. If symptomatic or with a concerning history, the interpretation warrants great care and other differential diagnosis should be considered [olivera, herneth2, kloiber]. 


\section{Infectious}

\section{C.1. Septic arthritis}

Septic arthritis is a joint infection that can occur by direct spread of contiguous osteomyelitis, hematogenous spread, or directly after penetrating trauma. It is a surgical emergency as it can have a rapidly deteriorating course with destruction of the joint. Septic arthritis in children is usually monoarticular; the hip is the most commonly affected joint in children under 10 years of age. Clinically, children are ill, with fever and severe joint pain. Unilaterally joint effusion is present in nearly all cases. Increased pressure within the joint may lead to ischemia or infarction of the femoral head epiphysis. Absence of fluid excludes septic arthritis. Treatment is surgical with urgent debridement and IV antibiotics [buchman].

Ultrasound is the first imaging modality of choice, both in detection of a joint effusion and guiding needle aspiration. When an effusion is present, the joint capsule becomes convex instead of concave, the anterior joint recess normally measures 2 to $5 \mathrm{~mm}$ from the outer margin of the capsule to the femoral neck cortex whereas the abnormal joint capsule should be at least $2 \mathrm{~mm}$ thicker than the contralateral normal side [grisson]. Effusion size and echotexture are not predictive of infection. A thickened synovium can also be seen. Increased flow at Doppler Ultrasound is more characteristic of a septic joint, but findings can be normal. Joint aspiration is both diagnostic and therapeutic, relieving intracapsular pressure, and is necessary to differentiate septic from transient synovitis [sarwar].

Conventional radiography may detect a large effusion and soft tissue swelling in the early stage, and in more advanced stages, may serve to exclude bone involvement, seen as cortical destruction, periosteal reaction, and subluxation [sarwar].

MRI may be helpful to evaluate the extent of the disease, and to detect concomitant osteomyelitis. MRI findings however are nonspecific, demonstrating joint effusion, synovial inflammation and enhancement after intravenous gadolinium administration, but no distinction between septic and transient synovitis can be made. The presence of periarticular BME and decreased perfusion of the femoral head after contrast administration favors septic arthritis [kwack]. More extensive bone marrow changes raise suspicion of osteomyelitis [rosenfeld].

Bone scintigraphy findings are nonspecific, showing increased periarticular uptake.

\section{C.2. Osteomyelitis/CRMO}

\section{C.2.1. Osteomyelitis}

Osteomyelitis of the hip is uncommon. It can occur at any age, generally in children 1-16 years old, and is usually caused by hematogenous spread of infection. Generally, the metaphysis is involved, transphyseal spread to the epiphysis and joint is rare but can occur in children younger than 18 months, or in older children with closed growth plates [schmit, blickman]. Patients present with hip pain and fever, passive range of motion is often preserved. Blood culture results are positive in fewer than half of cases. A subtype of osteomyelitis typically seen in children is Brodie's abscess, which is a subacute osteomyelitis with intraosseous abscess formation. Pain can be the only symptom, and fever and inflammatory 
markers can be abscent. Osteomyelitis is treated with IV antibiotics. Abscesses should be drained (imaging guided), and devitalized bone surgical debrided [klein, schmith].

The first imaging modality of choice is conventional radiography, but CR has limited sensitivity in early stages. Osseous destruction is evident only 10-14 days after the initial infection. In low-grade osteomyelitis, subtle bone resorption / a lytic lesion at a metaphysis may be seen, as in more advanced stages, extensive soft tissue edema, distortion of fat planes, lytic bone destruction and periosteal formation are seen [sarwar, khanna, blickman]. In case of Brodie's abscess, a sharp lytic lesion with our without sclerotic rim is seen in the metaphysis or in the epiphysis in young children.

$M R I$ is indicated when symptoms are localized to the pelvis and radiographs are normal. MRI is more specific in detection of lesions, but is also effective in depicting complications such as soft tissue or intraosseous abscesses and necrotic bone [sarwar]. MRI shows bone marrow edema as T2 hyperintense signal in the metaphysis, with post contrast enhancement of bone marrow, abscess margins, periosteum and adjacent soft tissue collections [schmit].

Bone scintigraphy can be performed for detection of radiographically occult disease and for assessment of multifocal involvement, although more recently, whole body MRI is performed increasingly [von kale, villani].

\section{C.2.2. Chronic recurrent multifocal osteomyelitis}

Chronic recurrent multifocal osteomyelitis (CRMO) is an autoinflammatory disorder of children and young adults, that is characterized by nonbacterial osteomyelitis. It is seen more commonly in girls than boys, the peak age of incidence is around 10 years. Multiple sites of osteomyelitis are present concomitantly, typically in the metaphyses of long bones and the medial clavicles. Other common sites are the vertebral bodies, pelvis, ribs, and mandible. CRMO is often bilateral and multifocal at presentation [huber].

The imaging evaluation of CRMO should start with conventional radiography of the symptomatic sites. If CR is negative, but significant clinical symptoms are present, further evaluation with MRI should be considered to evaluate for marrow edema. When the diagnosis of CRMO is considered based on this findings, further evaluation of the whole body is suggested to identify multifocal lesions that may be clinically asymptomatic [khanna]. Bone scintigraphy can be used to for this whole-body evaluation, but recently, whole-body MRI is being increasingly used, as MRI has no ionizing radiation [khanna].

\section{Inflammatory}

\section{D.1. Transient synovitis}

Transient or toxic synovitis is the most common nontraumatic hip disorder in toddlers and children up to 10 years of age. Boys are more commonly affected. It is a self-limiting aseptic inflammation of the hip, with unknown cause, but often seen after a viral infection. Allergic and traumatic causes have been suggested as well. Generally, children are not sick, and may show limb, pain and limited motion of the 
hip. Treatment is conservative, with rest and analgesics, and resolves usually in 3-10 days [zucker, miralles, do].

Imaging is not strictly necessary, but US might be the initial imaging study performed to demonstrate the presence of joint fluid, or to guide joint aspiration, which is the reference standard for differentiation with septic arthritis [sarwar, miralles]. Conventional radiography is not indicated, and only used to exclude other etiologies such as fracture, SCFE or AN. It may show atypical joint space widening, but are most often normal. MRI is not indicated, and may show joint effusion and synovial inflammation. MRI findings are non-specific and cannot differentiate with septic arthritis, although the presence of BME or osteomyelitis is suspicious and favors septic arthritis (cfr. 'Septic Arthritis') [sarwar, kwack].

\section{D.2. Juvenile Idiopathic Arthritis}

Juvenile Idiopathic Arthritis (JIA)is the most common form of arthritis in children and adolescents. JIA is an autoimmune, non-infective, inflammatory joint disease in children less than 16 years of age with arthritis that persists for more than 6 weeks [ravelli]. JIA recently is divided into 7 subtypes, according to the International League of Associations for Rheumatology (ILAR) classification [petty]. The large joints are mainly affected, with hip involvement in about $30-50 \%$ of cases, which is commonly bilateral. Clinically, morning stiffness is seen, improving with exercise, groin pain, or referred pain to the knee. Treatment is specialized in a pediatric rheumatology department, and includes physical therapy, antiinflammatory agents, corticosteroids and even disease-modifying antirheumatic drugs (DMARD) or even aggressive therapy with anti-tumor necrosis factor (anti-TNF) agents [ravelli].

Conventional radiography the first imaging modality, although often normal in early stages, sometimes soft tissue swelling, joint effusion, peri-articular osteopenia can be seen. Later stages may show joint space narrowing, periosteal reaction, accelerated bone maturation, erosions and eventually ankylosis [buchmann, sheybani, williams].

Ultrasound can depict joint effusion, synovial thickening, and is excellent for guiding aspiration. Doppler ultrasound can evaluate hyperemia, which correlates with disease activity [sheybani].

Recently MRI is increasingly used as it can detect early changes long before radiographic changes become evident. MRI can show joint effusion, bone marrow and soft tissue edema, synovial proliferation, and cartilaginous and bony erosions [sheybani, eshed].

\section{E. Neoplastic}

Bone tumors or tumorlike lesions of the hip in children are rare. There is often a delay in diagnosis because symptoms are frequently nonspecific, some are detected incidentally or when a pathologic fracture occurs [zucker]. Most (more than 4/5) neoplastic lesions are benign, malignant tumors of the hip are very rare. The most common benign lesions of the hip are simple bone cyst, osteoid osteoma, eosinophilic granuloma, aneurysmal bone cyst, fibrous dysplasia and osteochondromas. The most frequently seen malignant tumors of the hip are Ewing sarcoma, osteosarcoma, lymphoma, leukemia or metastasis [Ruggieri, zucker]. 
Detailed discussion of this lesions is beyond the scope of this article.

\section{Conclusion}

Hip disorders are common in children. The age of the child, history, clinical examination and in some cases laboratory findings are essential to narrow down the differential diagnosis. Together with the appropriate imaging modality findings, the accurate diagnosis can be found.

\section{References}

Zucker EJ, Lee EY, Restrepo R, Eisenberg RL. Hip disorders in children. AJR 2013; 201: 776-96.

Hubbard AM. Imaging of pediatric hip disorders. Radiol clin north am 2001; 39(4): 721-32.

Vanhoenacker FM, Jans L, Boutry N. The pediatric hip. In: Kramer J, Karantanas A, editors. MRI of the hip. Austria 2016. p. 2017-38.

Gough-Palmer A, McHugh K. Investigating hip pain in a well child. BMJ 2007; 334:1216.

Karami V, Zabihzadeh M, Shams N, Sarikhani S. "Evaluation of the Prevalence and Utility of Gonad Shielding in Pediatrics Undergoing Pelvic X-Ray." Int J Pediatr 2016: 4(11): 3735-40.

Frantzen MJ, Robben S, Postma AA, Zoetelief J, Wildeberger JE, Kemerink GJ. Gonad shielding in paediatric pelvic radiography: disadvantages prevail over benefit. Insights imaging 3(1):23-32.

$\mathrm{DDH}$

1.Bracken J, Tran T, Ditchfield M. Developmental dysplasia of the hip: controversies and current concepts. J Paediatr Child Health 2012; 48(11):963-72, quiz $972-3$.

Ortiz-Neira CL, Paolucci EO, Connon T. A metaanalysis of common risk factors associated with the diagnosis of developmental dysplasia of the hip in newborns. Eur J Radiol 2012; 81:e344-e351

Giannakopoulou C, Alagizakis A, Korakaki E, et al. Neonatal screening for developmental dysplasia of the hip on the maternity wards in Crete, Greece: correlation to risk factors.

Gill KG. Pediatric hip: pearls and pitfalls. Semin Musculoskelet

Radiol 2013; 17(3):328 - 38.

2.Bracken J, Ditchfield M. Ultrasonography in developmental

dysplasia of the hip: what have we learned? Pediatr Radiol 2012;

42(12):1418 - 31 . 
American Institute of Ultrasound in Medicine. AIUM Practice Guideline for the performance of an ultrasound examination for detection and assessment of developmental dysplasia of the hip. J Ultrasound Med 2013; 32:1307-17.

Graf R. Classification of hip joint dysplasia by means of sonography. Arch Orthop Trauma Surg 1984; 102:248-55.

Starr V, Ha BY. Imaging update on developmental dysplasia of the hip with the role of MRI. AJR 2014 Dec;203(6):1324-35. doi: 10.2214/AJR.13.12449.

El Ferzli J, Aburamara S, Eurin D, Le Dosseur P, Dacher JN. Anterior axial ultrasound in monitoring infants with Pavlik harness. Eur Radiol 2004; 14:73-77.

Barnewolt CE, Jaramillo D, Taylor GA, Dunning PS. Correlation of contrast-enhanced power Doppler sonography and conventional angiography of abduction-induced hip ischemia in piglets. AJR 2003; 180:1731-5.

2. Starr V, Ha B. Developmental dysplasia of the hip (DDH). In: Daldrup-Link HE, Newman B, eds. Pearls and pitfalls in pediatric imaging, variants and other difficult diagnoses. New York, NY: Cambridge University Press, 2013:335-8.

Donnelly LF. Developmental dysplasia of the hip. In: Donnelly LF. Pediatric imaging: the fundamentals. Philadelphia, PA: Elsevier, 2009:188-91.

Beltran LS, Rosenberg ZS, Mayo JD, et al. Imaging evaluation of developmental hip dysplasia in the young adult. AJR 2013; 200:1077-88.

Chin MS, Betz BW, Halanski MA. Comparison of hip reduction using magnetic resonance imaging or computed tomography in hip dysplasia. J Pediatr Orthop 2011; 31:525-529

McNally EG, Tasker A, Benson MK. MRI after operative reduction for developmental dysplasia of the hip. J Bone Joint Surg Br 1997; 79:724-726

Conroy E, Sproule J, Timlin M, McManus F. Axial STIR MRI: a faster method for confirming femoral head reduction in DDH. J Child Orthop 2009; 3:223-227

Laor T, Roy DR, Mehlman CT. Limited magnetic resonance imaging examination after surgical reduction of developmental dysplasia of the hip. J Pediatr Orthop 2000; 20:572-574

Jaramillo D, Villegas-Medina O, Laor T, Shapiro F, Millis MB. Gadolinium-enhanced MR imaging of pediatric patients after reduction of dysplastic hips: assessment of femoral head position, factors impeding reduction, and femoral head ischemia. AJR 1998; 170:1633-1637

Tiderius C, Jaramillo D, Connolly $\mathrm{S}$, et al. Postclosed reduction perfusion magnetic resonance imaging as a predictor of avascular necrosis in developmental hip dysplasia: a preliminary report. J Pediatr Orthop $2009 ; 29: 14-20$ 
Kim HT, Kim JI, Yoo CL. Diagnosing childhood acetabular dysplasia using the lateral margin of the sourcil. J Pediatr Orthop 2000; 20:709-717

Pirpiris M, Payman KR, Otsuka NY. The assessment of acetabular index: is there still a place for plain radiography? J Pediatr Orthop 2006; 26:310-315

Ray PS, Redden JF, Ward D. Closed reduction of late-presented DDH: MRI view of remodelling. J Bone Joint Surg Br 2003; 85-B(suppl II):109

Li LY, Zhang LJ, Li QW, et al. Development of the osseous and cartilaginous acetabular index in normal children and those with developmental dysplasia of the hip: a cross sectional study using MRI. J Bone Joint Surg Br 2012; 94:1625-1631

Perthes:

Houghton KM. Review for the generalist: evaluation of pediatric hip pain. Pediatr Rheumatol Online J 2009; 7:10. DOI: 10.1186/1546- 0096-7-10.

De Smet E, Vanhoenacker F, Petre C, Bosmans JL, Verstraete KL. Enfant presentant une tete femoral aplatie. Ortho-rhumato 2013;10(2):23 - 25.

Kocher MS, Tucker R. Pediatric athlete hip disorders. Clin Sports Med 2006; 25(2):241 - 253.

Dillman JR, Hernandez RJ. MRI of Legg-Calvé-Perthes Disease. AJR 2009; 193:1394-1407. DOI:10.2214/AJR.09.2444

Herring JA, Neustadt JB, Williams JJ, Early JS, Browne RH. The lateral pillar classification of Legg-CalvéPerthes disease. J Pediatr Orthop. 1992 Mar-Apr;12(2):143-50.

Cheng JC, Lam TP, Ng BK. Prognosis and prognostic factors of LeggCalve-Perthes disease. J Pediatr Orthop 2011;31(2, Suppl): S147-S151.

Coxa vara:

Bos CF, Sakkers RJ, Bloem JL et al. Histological, biochemical and MR imaging studies of the growth plate in congenital coxa vara. J Pediatr Orthop 1989; 9:660.

Serafin J, Szulc W. Coxa vara infantum, hip growth. Clin Orthop 1991; 272:103.

Desai SS, Johnson LO. Long-term results of valgus osteotomy for congenital coxa vara. Clin Orthop 1993; 294:204.

Günther CM, Komm M, Jansson V, Heimkes B. Midterm results after subtrochanteric end-to-side valgization osteotomy in severe infantile coxa vara. J Pediatr Orthop 2013: 33(4):353-60. 
Neuromuscular hip/coxa valga

Scrutton D, Baird G. Surveillance measures of the hips of children with bilateral cerebral palsy. Arch Dis Child 1997; 76:381.

Chang $\mathrm{CH}$, Kuo KN, Wang CJ, Chen YY, Cheng HY, Kao HK. Acetabular deficiency in spastic hip subluxation. J Pediatr Orthop 2011; 31(6):648-54.

Khalife R, Ghanem I, El Hage S, Dagher F, Kharrat K. Risk of recurrent dislocation and avascular necrosis after proximal femoral varus osteotomy in children with cerebral palsy. J Pediatr Orthop B 2010;19(1):32-37.

Hagglund G, Lauge-Pedersen H, Persson M. Radiologic threshold values for hip screening in cerebral palsy. J Pediatr Orthop 2007; 1(1):43-47.

Reimers J. The Stability of the Hip in Children: A Radiological Study of the Results of Muscle Surgery in Cerebral Palsy. Acta Orthop Scand suppl. 1980; 51(184):1-100. DOI: 10.3109/ort.1980.51.suppl-184.01

Terjesen T. Residual hip dysplasia as a risk factor for osteoarthritis in 45 years follow-up of late-detected hip dislocation. J Child Orthop 2011;5(6):425-431.

Scrutton D, Baird G, Smeeton N. Hip dysplasia in bilateral cerebral palsy: incidence and natural history in children aged 18 months to 5 years. Dev Med Child Neurol 2001;43(9):586-600.

\section{SCFE}

Gholve PA, Cameron DB, Millis MB. Slipped capital femoral epiphysis update. Current opinion in pediatrics 2009; 21:39-45. 45.

Tins B, Cassar-Pullicino V, McCall I. Slipped upper femoral epiphysis: imaging of complications after treatment. Clin Radiol 2008; 63(1):27 - 40.

Lubicky JP. Chondrolysis in avascular necrosis: complications. Slipped capital femoral epiphysis. J Pediatr Orthop 1996; 5:162.

Tins B, Cassar-Pullicino V, McCall I. The role of pre-treatment MRI in established cases of slipped capital femoral epiphysis. Eur J Radiol 2009; 70(3):570 - 8.

fractures

Johnson KJ, Bache E. Imaging in pediatric skeletal trauma. Techniques and applications. Springer 2008; 3 -10 .

Boutry N, Amzallag E, Nectoux E, Fron D, Herbaux B. Traumatismes du bassin et de la hance chez I'enfant. In : Cotten A, Vande berg B. 15émes mises au point en imagerie osteo-articulaire. Lille, Bruxelles 2015.

Watts HG. Fractures of the pelvis in children. Orthop Clin North Am 1976; 7(3):615 -624.

Torode I, Zieg D. Pelvic fractures in children. J Pediatr Orthop 1985; 5(1):76 - 84. 
Quick T.J., Eastwood D.M. Pediatric fractures and dislocations of the hip and pelvis. Clin Orthop Relat Res. 2005;(432):87-96.

Bali K, Sudesh P, Patel S, Kumar V, Saini U, Dhillon MS. Pediatric femoral neck fractures: our 10 years of experience. Clin Orthop Surg 2011; 3(4):302 - 308.

Vigler M, Weigl D, Schwarz M, Ben-Itzhak I, Salai M, Bar-On E. Subtrochanteric femoral fractures due to simple bone cysts in children. J Pediatr Orthop B 2006; 15(6):439 - 442.

Karantanas AH. Hip. Sports injuries in children and adolescents. Springer 2011; $163-189$.

Behrens SB, Deren ME, Matson A, Fadale PD, Monchik KO. Stress fractures of the pelvis and legs in athletes: a review. Sports Health 2013; 5(2):165 - 174.

Campbell SE, Fajardo RS. Imaging of stress injuries of the pelvis. Semin Musculoskelet Radiol 2008; $12(1): 62-71$.

Arendt EA, Griffiths HJ. The use of MR imaging in the assessment and clinical management of stress reactions of bone in high-performance athletes. Clin Sports Med 1997; 16(2):291-306.

Avulsion injuries

Rossi F, Dragoni S. Acute avulsion fractures of the pelvis in adolescent competitive athletes: prevalence, location and sports distribution of 203 cases collected. Skeletal Radiol 2001; 30(3):127 - 131.

Vandervliet EJ, Vanhoenacker FM, Snoeckx A, Gielen JL, Van Dyck P, Parizel PM. Sports-related acute and chronic avulsion injuries in children and adolescents with special emphasis on tennis. Br J Sports Med 2007; 41(11):827-831.

Kovacevic D, Mariscalco M, Goodwin RC. Injuries about the hip in the adolescent athlete. Sports Med Arthrosc 2011; 19:64.

Stevens MA, El-Khoury GY, Kathol MH, Brandser EA, Chow S. Imaging features of avulsion injuries. Radiographics. 1999; 19(3):655 - 672. 7.

McKinney BI, Nelson C, Carrion W. Apophyseal avulsion fractures of the hip and pelvis. Orthopedics $2009 ; 32(1): 42-48$.

Meyers AB, Laor T, Zbojniewicz AM, Anton CG. MRI of radiographicallly occult ischial apophyseal avulsions. Pediatr radiol 2012; 42(11): 1357-63.

Vansevenant M, Vanhoenacker F, Verstraete KL. Ischiofemoral impingement in young patients. EPOS poster European Society of Musculoskeletal Radiology 2014; DOI: http://dx.doi.

org/10.1594/essr2014/P-0023.

Laor T. Hip and groin pain in adolescents. Pediatr Radiol 2010; 40(4): 461-7. 
Ischiopubic synchondrosis

Karantanas AH. Hip. Sports injuries in children and adolescents. Springer 2011; 42-43.

Oliveira F. Differential diagnosis in painful ischiopubic synchondrosis (IPS): a case report. lowa Orthop J 2010; 30:195 - 200.

Herneth AM, Philipp MO, Pretterklieber ML, Balassy C, Winkelbauer FW, Beaulieu CF. Asymmetric closure of ischiopubic synchondrosis in pediatric patients: correlation with foot dominance. AJR Am J Roentgenol 2004; 182(2):361 - 365.

2 Herneth AM, Trattnig S, Bader TR et al. MR imaging of the ischiopubic synchondrosis. Magnetic Resonance Imaging $2000 ; 18: 519-524$.

Kloiber R, Udjus K, Mclntyre W et-al. The scintigraphic and radiographic appearance of the ischiopubic synchondroses in normal children and in osteomyelitis. Pediatr Radiol. 1988;18 (1): 57-61.

Septic arthritis

Buchmann RF, Jaramillo D. Imaging of articular disorders in children. Radiol Clin North Am 2004;42(1):151-168.

Grisson L, Harcke H. Pediatric musculoskeletal ultrasound. In: Rumac C, Wilson S Charboneau et al. Diagnostic ultrasound $3^{\text {rd }}$ ed. St Louis, MO: Elsevier Mosby; 2005: 2048.

Sarwar ZU, DeFlorio R, Catanzano TM. Imaging of nontraumatic acute hip pain in children: multimodality approach with attention to the reduction of medical radiation exposure. Semin Ultrasound CT MR 2014; 35:394.

Kwack KS, Cho JH, Lee JH, et al. Septic arthritis versus transient synovitis of the hip: gadoliniumenhanced MRI finding of decreased perfusion at the femoral epiphysis. AJR Am J Roentgenol 2007; 189:437.

Rosenfeld S, Bernstein DT, Daram S, et al. Predicting the Presence of Adjacent Infections in Septic Arthritis in Children. J Pediatr Orthop 2016; 36:70.

Osteomyelitis

Klein JD, Leach KA. Pediatric pelvic osteomyelitis. Clin Pediatr (Phila). 2007 Nov. 46(9):787-90.

Schmit P, Glorion C. Osteomyelitis in infants and children. Eur Radiol. 2004 Mar. 14 Suppl 4:L44-54.

Khanna G, Sato TSP, Ferguson P. Imaging of Chronic Recurrent Multifocal Osteomyelitis. RadioGraphics 2009; 29:1159-1177.

Blickman JG, van Die CE, de Rooy JW. Current imaging concepts in pediatric osteomyelitis. Eur Radiol. 2004 Mar. 14 Suppl 4:L55-64. 
von Kalle T, Heim N, Hospach T, Langendörfer M, Winkler P, Stuber T. Typical patterns of bone involvement in whole-body MRI of patients with chronic recurrent multifocal osteomyelitis (CRMO). Rofo. 2013 Jul;185(7):655-61. doi: 10.1055/s-0033-1335283.

Villani MF, Tanturri de Horatio L, Garganese MC, Casazza I, Savelli S, Pardeo M, Messia V, De Benedetti $\mathrm{F}$, Insalaco A. Whole-Body MRI versus bone scintigraphy: which is the best diagnostic tool in patients with chronic recurrent multifocal osteomyelitis (CRMO)? Pediatr Rheumatol Online J. 2015; 13(Suppl 1): P58. Published online 2015 Sep 28. doi: 10.1186/1546-0096-13-S1-P58

Huber AM, Lam PY, Duffy CM, et al. Chronic recurrent multifocal osteomyelitis: clinical outcomes after more than five years of follow-up. J Pediatr.2002; $141: 198$-203

Fritz J, Tzaribachev N, Kummerl-Deschner J, Claussen CD, Carrino JA, Horger M. Whole-body magnetic resonance imaging of children with new-onset chronic recurrent multifocal osteomyelitis: description and comparison with laboratory and clinical data [abstr]. In: Radiological Society of North America scientific assembly and annual meeting program. Oak Brook, Ill: Radiological Society of North America, 2008; 1034.

Transient synovitis

Miralles M, Gonzalez G, Pulpeiro JR, Millán JM, Gordillo I, Serrano C, Olcoz F, Martinez A. Sonography of the painful hip in children: 500 consecutive cases. AJR.1989;152 (3): 579-82.

Do TT. Transient synovitis as a cause of painful limps in children. Curr. Opin. Pediatr. 2000;12 (1): 48-51.

JIA

Ravelli A, Martini A. Juvenile Idiopathic Arthritis. Lancet 2007;369:767-78.

Petty RE, Southwood TR, Manners P, Baum J, Glass DN, Goldenberg J et al. International League of Association for Rheumatology Classification of juvenile idiopathic arthritis: second revision. Edmonton. 2001. J Rheumatol 2004;31:390-2.

Sheybani EF, Khanna G, White AJ et-al. Imaging of juvenile idiopathic arthritis: a multimodality approach. Radiographics. 2013;33 (5): 1253-73.

Williams RA, Ansell BM. Radiological findings in seropositive juvenile chronic arthritis (juvenile rheumatoid arthritis) with particular reference to progression. Ann. Rheum. Dis. 1985;44 (10): 685-93.

Eshed I, Bollow M, McGonagle DG, Tan AL, Althoff CE, Asbach P et al. MRI of enthesitis of the appendicular skeleton in spondyloarthritis. Ann Rheum Dis 2007;66:1553-9.

Neoplastic

Ruggieri P, Angelini A, Montalti M, et al. Tumours and tumourlike lesions of the hip in the paediatric age: a review of the Rizzoli experience. Hip Int 2009; 19 Suppl 6:S35. 
